Введение. Разработан режим фракизонирования дозы излучения при химиолучевом лечении для снижения частоты и тяжести ранних и поздних лучевых реакичий нормальных тканей.

Цель исследования. Уменьшить частоту и выраженность ранних и поздних лучевых реакций нормальныхх тканей у пациентов с плоскоклеточным раком слизистой оболочки полости рта, языка и глотки III и IVA, В стадий путем применения разработанного режима динамического ускоренного гиперфракционирования дозы излучения с сопутствуюшим бустом при химиолучевом лечении.

Материал и методы. Проанализированы данные 280 пациентов с плоскоклеточнылм раком слизистой оболочки полости рта, языка, рото- и гортаноглотки III и IVA, В стадий. Пациентам основной группы проводилась лучевая терапия в нетрадиционном режиме фракционирования. В контрольной группе использовался конвенциональный режим фракциионирования дозы излучения. Проводилась сравнительная оченка непосредственной эффективности лучевой терапии с классическим и нетрадиционным режимами фракционирования.

Результаты исследования. В основной группе преобладала 2-я степень (63,6\%) ранних лучевых реакиий слизистых оболочек, в контрольной группе - 3 (47,5\%) и 2 (41,6\%) степень реакций (p<0,001). Ранние лучевые реакции кожи в основной группе в 53\% случаев встречались в 1-й степени, а в контрольной группе чаше $(59,9 \%)$ развивались реакциии кожи 2-й степени (p<0,001). Поздние лучевые реакции кожи 1-й степени чаше встречались в основной группе - 75\% по сравнению с контрольной группой - 50\% (p<0,001).

Bblводы. B группе с нетрадиционным режимом фракционирования получено статистически значимое снижение частоты и степени лучевых реакций.

Ключевые слова: рак орофарингеальной зоны, ранние и поздние лучевые реакции, режим нетрадиционного фракционирования.

\section{Введение}

Традиционным методом лечения пациентов с местно-распространенным плоскоклеточным раком слизистой оболочки полости рта, языка и глотки остается лучевая терапия. По результатам многочисленных клинических испытаний уже доказана целесообразность лучевой терапии с одновременным введением цисплатина на ее фоне $[1,2,3,4]$. Вместе с тем сравнительно низкие показатели локо-регионального контроля опухоли и выраженные ранние лучевые реакции и поздние лучевые повреждения нормальных тканей при конвенциональной лучевой терапии диктуют необходимость разработки новых, радиобиологически обоснованных вариантов фракционирования дозы излучения. Наиболее эффективным при лучевой терапии злокачественных опухолей орофарингеальной зоны считается использование режимов гиперфракционирования и ускоренного фракционирования. $[5,6,7,8]$.

Первый вариант фракционирования разработан для снижения вероятности развития ранних, и в особенности поздних лучевых повреждений нормальных тканей за счет подведения к объему облучения разовых очаговых доз, меньших по сравнению с дозами при стандартном курсе лучевой терапии, а также за счет шестичасовых перерывов между дневными фракциями, позволяющих клеткам нормальных тканей восстановиться от сублетальных повреждений за этот период [9]. Длительность таких курсов сопоставима по времени облучения при конвенциональ- ной радиотерапии, что существенно не сказывается на частоте возникновения и выраженности поздних лучевых повреждений, формирующихся в медленно обновляющихся тканях. Однако полная доза, требуемая для контроля опухоли, должна быть увеличена при увеличении числа фракций, так как механизм репарации повреждений проявляется не только в нормальных тканях, но и в опухоли.

Многочисленные экспериментальные и клинические исследования подтверждают зависимость эффекта уменьшения клеточной выживаемости при увеличении дозы за фракцию, что дает возможность более быстрой остановки роста опухоли, а также при использовании режимов дневного дробления дозы или динамического фракционирования с запланированным изменением разовых очаговых доз в процессе лечения. Эти шаги предпринимаются для уменьшения продолжительности курса лучевой терапии, так как оптимальным для опухолей головы и шеи являются режимы облучения общей длительностью от 4 до 6 недель с относительным уровнем выживших клеток опухоли $10^{-11}$ [10]. Объясняется это в первую очередь процессом ускоренной опухолевой репопуляции, возникающей на четвертой неделе облучения, для преодоления которой необходимо сокращать время облучения или увеличивать общую дозу для уничтожения новой популяции опухолевых клеток.

Серьезность повреждения нормальных тканей зависит от дозы и ее распределения во времени и пространстве. Эффекты облучения 
становятся тяжелыми с увеличением дозы излучения и с увеличением поля облучения (объемный эффект). Повреждения нормальных тканей усугубляются с увеличением дозы за фракцию при сохранении полной дозы, могут проявляться сильнее при уменьшении общего времени облучения. В этом случае лучше использовать дневное дробление дозы с интервалом не менее 6 часов для эффективной репарации радиационных повреждений нормальных тканей. Кроме того, при ускоренных режимах фракционирования с уменьшением общего времени лечения ранние лучевые реакции нормальных тканей III-IV степени не успевают развиться, что дает возможность подвести к опухоли полную запланированную дозу без вынужденных перерывов на ликвидацию лучевых осложнений.

В нашем исследовании был разработан метод лучевой терапии, сочетающий в себе наиболее эффективный режим фракционирования дозы излучения для опухолей орофарингеальной зоны - это режим гиперфракционирования, который динамически менялся во время непрерывного курса на вариант лучевой терапии с сопутствующим бустом для сокращения общего времени лечения.

Цель исследования: уменьшить частоту и выраженность ранних и поздних лучевых реакций нормальных тканей у пациентов с плоскоклеточным раком слизистой оболочки полости рта, языка и глотки III и IVA, В стадий путем применения разработанного режима динамического ускоренного гиперфракционирования дозы излучения с сопутствующим бустом при химиолучевом лечении.

\section{Материал и методы}

В проспективное рандомизированное исследование были включены 280 пациентов с диагнозом местно-распространенного рака слизистой оболочки полости рта, языка, ротоглотки и гортаноглотки III, IVA и IVB стадий. Во всех случаях диагноз был верифицирован морфологически и соответствовал по гистологической классификации ВО3 плоскоклеточному раку. Все пациенты, включенные в исследование, были мужчинами.

После рандомизации в группу лучевой терапии с нетрадиционным режимом фракционирования дозы излучения (основная группа) вошли 139 пациентов в возрасте от 40 до 69 лет. В контрольную группу лучевой терапии с классическим режимом фракционирования был включен 141 пациент в возрасте от 35 до 70 лет. Средний возраст пациентов как в основной, так и в контрольной группах составил 56 лет $(\mathrm{SD}=7)$.

При сравнении групп исследования по стадии заболевания, категориям Т и $\mathrm{N}$, степени дифференцировки опухоли, локализации рака в орофарингеальной зоне не выявлено статистически значимых раз- личий. Анализируемые группы пациентов полностью сопоставимы (табл. 1).

Пациентам основной группы дистанционная конформная лучевая терапия проводилась в два этапа без перерыва в течение 5 недель. На первом этапе (3 недели) использовался режим гиперфракционирования с подведением разовой очаговой дозы (РОД) 1,2 Грей дважды в день на опухоль и зоны субклинического распространения (регионарные лимфатические узлы). На втором этапе (2 недели) применялся режим ускоренного фракционирования с сопутствующим бустом и подведением РОД 1,8 Грей в первую дневную фракцию на вышеописанную зону и РОД 1,6 Грей локально на опухоль и метастатические лимфатические узлы во вторую фракцию. В 1 и 22-й дни облучения вводился цисплатин в дозе $100 \mathrm{мг} / \mathrm{M}^{2}$ внутривенно. Суммарная очаговая доза (СОД) за весь курс лечения составила 54 Грей на зоны субклинического распространения и 70 Грей на опухолевые очаги.

Пациентам контрольной группы проводилась дистанционная конформная лучевая терапия в классическом режиме фракционирования в течение 7 недель, 1 раз в день, 5 раз в неделю, РОД 2 Грей до СОД 70 Грей на первичный очаг и в СОД 50-60 Грей на регионарные лимфатические узлы с введением цисплатина в 1-й, 22-й и 43-й

Таблица 1. - Сравнительная характеристика пациентов с местно-распространенным раком полости рта, языка и глотки двух групп исследования

\begin{tabular}{|c|c|c|c|}
\hline Показатель & $\begin{array}{c}\text { Контрольная } \\
\text { группа (1), } \\
\text { n=141 }\end{array}$ & $\begin{array}{c}\text { Основная } \\
\text { группа }(2), n=139\end{array}$ & $\mathrm{p}$ \\
\hline \multicolumn{4}{|c|}{ Категория T, n (\%) } \\
\hline $\mathrm{T} 1$ & $7(5)$ & $2(1,4)$ & \multirow{4}{*}{0,211} \\
\hline $\mathrm{T} 2$ & $22(15,6)$ & $15(10,8)$ & \\
\hline T3 & $94(66,7)$ & $102(73,4)$ & \\
\hline T4 & $18(12,7)$ & $20(14,4)$ & \\
\hline \multicolumn{4}{|c|}{ Категория N, n (\%) } \\
\hline N0 & $44(31,3)$ & $53(38,0)$ & \multirow{4}{*}{0,089} \\
\hline N1 & $57(40,4)$ & $39(28,1)$ & \\
\hline $\mathrm{N} 2$ & $37(26,2)$ & $39(28,1)$ & \\
\hline N3 & $3(2,1)$ & $8(5,8)$ & \\
\hline \multicolumn{4}{|c|}{ Стадия, n (\%) } \\
\hline III & $91(64,5)$ & $75(54)$ & \multirow[t]{2}{*}{0,093} \\
\hline IV & $50(35,5)$ & $64(46)$ & \\
\hline \multicolumn{4}{|c|}{ Степень дифференцировки G, n (\%) } \\
\hline G1 & $20(15,2)$ & $20(16,2)$ & \multirow{3}{*}{0,713} \\
\hline G2 & $105(79,5)$ & $99(80,5)$ & \\
\hline G3 & $7(5,3)$ & $4(3,3)$ & \\
\hline \multicolumn{4}{|c|}{ Локализация первичной опухоли, n (\%) } \\
\hline Ротоглотка $(\mathrm{C} 1, \mathrm{C} 9, \mathrm{C} 10)$ & $70(49,6)$ & $67(48.2)$ & \multirow{4}{*}{0,618} \\
\hline Полости рта (C3, C4, C6) & $14(9,9)$ & $21(15.1)$ & \\
\hline Язык (C2) & $8(5,7)$ & $7(5)$ & \\
\hline $\begin{array}{l}\text { Гортаноглотка } \\
\text { С13) }\end{array}$ & $49(34,8)$ & $44(31.7)$ & \\
\hline
\end{tabular}


дни облучения.

Все расчеты проводились в статистическом пакете R, версия 3.3.1. Различия считались статистически значимыми при $\mathrm{p}<0,05$. Описательные характеристики групп исследования представлены частотами и процентами в группах, сравнение проводилось по точному критерию Фишера.

\section{Результаты и обсуждение}

С помощью линейно-квадратичной модели были рассчитаны биологически эффективная доза (BED) и суммарная эквивалентная доза (ESD).

Вероятность формирования выраженных поздних лучевых повреждений при разработанном методе лучевой терапии была ниже, чем у стандартного курса (BED на 7,1 Грей, ESD на 3,9 Грей). А вероятность развития ранних лучевых реакций была сопоставима со стандартным курсом (BED на 0,6 Грей и ESD на 0,5 Грей выше).

Математические расчеты по линейно-квадратичной модели для разработанного ускоренного режима фракционирования с дневным дроблением дозы согласуются с клиническими данными об острой и поздней токсичности.

Ранние и поздние лучевые реакции оценивались по шкале EORTC/RTOG: 0 степень - без изменений, 1-4 степени - разная выраженность лучевых реакций, 5 степень - смерть.

Изучались ранние лучевые реакции кожных покровов, слизистой оболочки полости рта и глотки, а также гематологическая токсичность (снижение показателей лейкоцитов в периферической крови).

Для изучения острой токсичности радиотерапии в расчет были взяты все пациенты, кроме 4 пациентов контрольной группы и 7 пациентов основной группы, не завершивших полный курс лучевой терапии. Фиксировалась максимальная степень выраженности лучевых реакций во время проведения лучевой терапии. У пациентов всех групп не выявлено ранних лучевых реакций V степени.

Слизистые оболочки полости рта и глотки были подвержены лучевым реакциям разной степени выраженности. Первая степень реакций проявлялась гиперемией слизистой и слабовыраженными болями в орофарингеальной зоне без необходимости применения анальгетиков. При второй степени развивался серозно-геморрагический отек с умеренными болями, требующими назначения анальгетиков. В дальнейшем появление выраженного серозно-геморрагического отека с сильными болями требовало применения наркотических средств, и это состояние соответствовало 3 степени ранних лучевых изменений. Изъязвление, некроз и кровотечение отражали 4 степень лучевых повреждений.

Ни в одной из групп не зафиксировано 0 степени реакций (рис.1).

Однако в основной группе преобладала
2 степень $(63,6 \%)$, а в контрольной группе - 3 степень $(47,5 \%)$ и 2 степень $(41,6 \%)$ реакций. Таким образом, группы были неоднородны $(<0,001)$ по степени выраженности ранних лучевых реакций слизистых оболочек.

Ранние лучевые реакции кожи 1-й степени клинически проявлялись очаговой слабовыраженной эритемой с эпиляцией, сухим шелушением и пониженной потливостью. Яркая эритема с очаговым влажным шелушением и умеренным отеком кожи наблюдалась при 2-й степени реакций. При 3-й степени влажный эпидермит приобретал сливной характер, а при 4-й степени развивался некроз кожи.

Менее выраженные ранние лучевые реакции кожи также значимо чаще наблюдались в основной группе с нетрадиционным режимом фракционирования дозы излучения (рис. 2).

По степени выраженности ранних лучевых реакций кожи группы были неоднородны $(<0,001)$. Анализ показал, что для контрольной группы характерны реакции 2-й степени (59,9\%), для основной - 1 и 2-й степени (соответственно, 53 и 44,7\%).

Гематологическая токсичность оценивалась по изменениям показателей лейкоцитов в периферической крови пациентов. Так, при 1-й степени количество лейкоцитов снижалось от 4,0 до

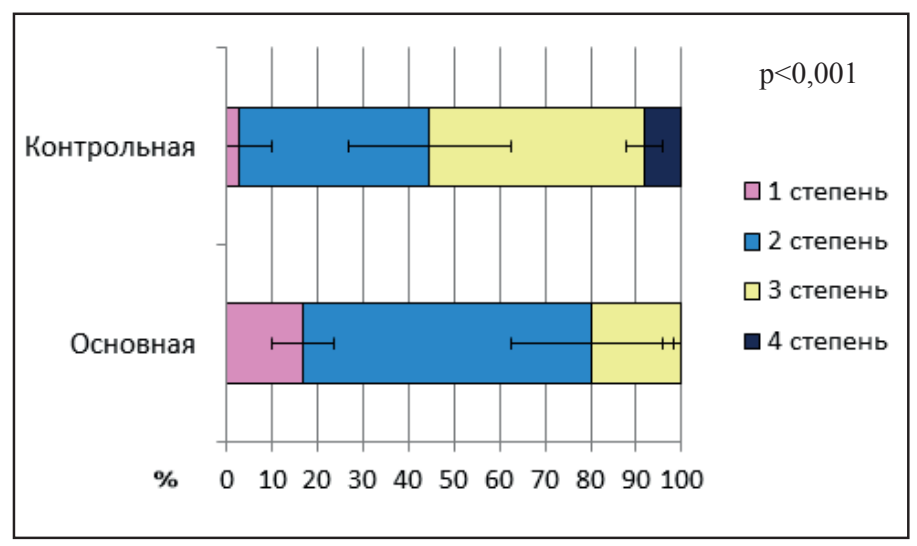

Рисунок 1. - Ранние лучевые реакции слизистых оболочек у пациентов контрольной и основной групп

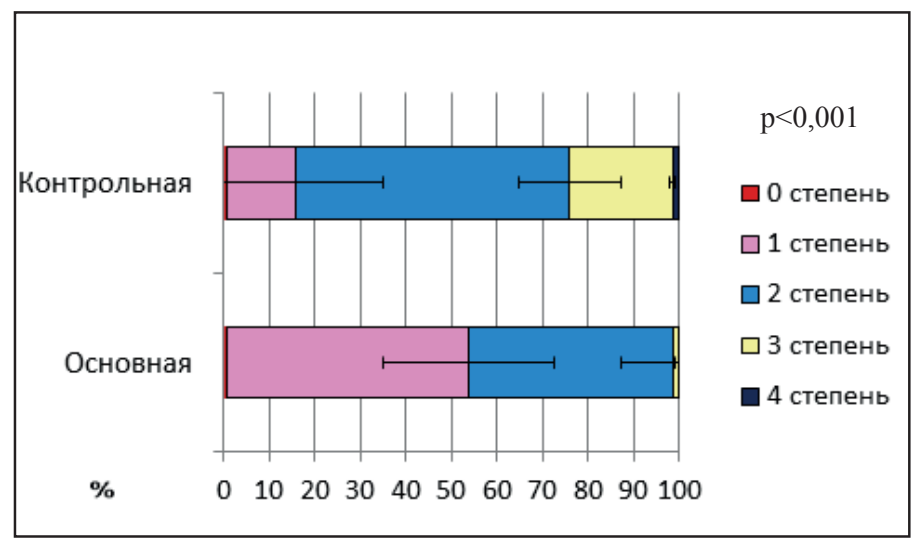

Рисунок 2. - Ранние лучевые реакции кожи у пациентов контрольной и основной групп 
$3,0 \times 10^{9} /$ л, при 2-й - от 3,0 до 2,0×109/л, при 3-й от 2,0 до $1,0 \times 10^{9} /$ л, а 4-я степень фиксировалась при показателях лейкоцитов ниже $1,0 \times 10^{9} /$ л.

Не наблюдалось изменений количества лейкоцитов периферической крови у 45,5\% пациентов группы с нетрадиционным режимом фракционирования и у 29,2\% пациентов контрольной группы, а 1-я степень гематологической токсичности встречалась у 48,5\% пациентов основной группы и у 45,3\% пациентов контрольной группы. Таким образом, по степени выраженности гематологической токсичности группы были однородны (рис. 3).

Поздние лучевые реакции кожи, подкожной клетчатки и слюнных желез изучались при контрольном осмотре через 3 месяца и более после окончания лучевой терапии. Фиксировалась максимальная степень выраженности лучевых повреждений. В данном исследовании не зафиксировано поздней токсичности V степени, приведшей к смерти пациента.

Не оценивались поздние лучевые реакции у 11 пациентов, не завершивших курс лучевой терапии, описанных ранее, а также у 12 пациентов контрольной группы и у 8 пациентов основной группы, т. к. они прожили менее трех месяцев после завершения лучевой терапии и не достигли срока оценки поздних лучевых реакций.

Поздние лучевые реакции кожи 1-й степени

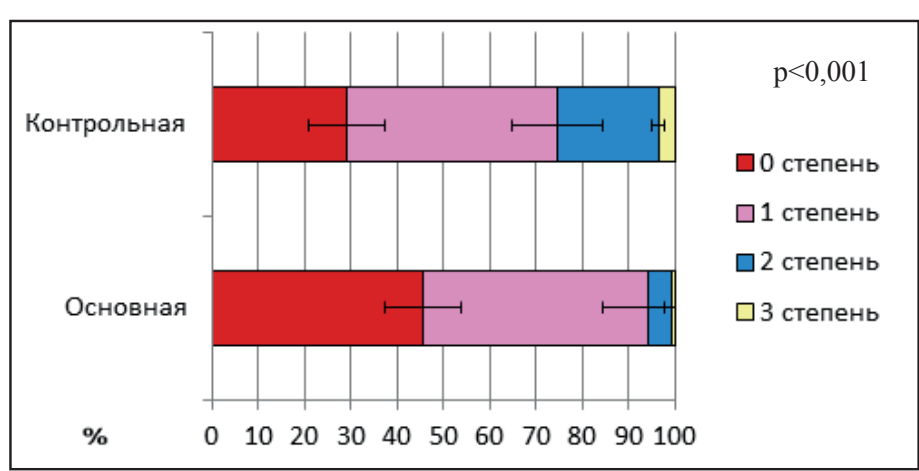

Рисунок 3. - Ранние лучевые реакции периферической крови (изменение показателей лейкоцитов) у пациентов контрольной и основной групп

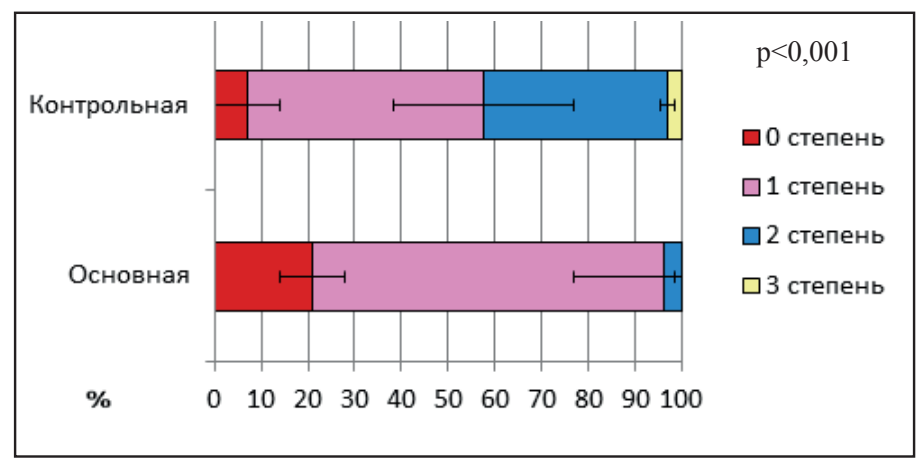

Рисунок 4. - Поздние лучевые повреждения кожи у пациентов контрольной и основной групп проявлялись пигментацией, слабовыраженной атрофией и выпадением волос в зоне предшествующего облучения. При умеренно выраженной очаговой атрофии с телеангиоэктазиями или признаками гипертрофического дерматита устанавливалась 2-я степень изменений. Выраженные телеангиоэктазии, атрофический или гипертрофический дерматит во всем поле облучения соответствовал 3-й степени. Появление лучевой язвы или радиоиндуцируемого рака относилось к 4-й степени повреждений.

В подкожной клетчатке при 1-й степени поздних лучевых реакций пальпировалось небольшое уплотнение тканей в поле облучения, при 2-й степени - умеренный смещаемый фиброз, клинически бессимптомный, при 3-й степени выраженный фиброз, а при 4-й степени - некроз.

Лучевые реакции слюнных желез 1-й степени проявлялись легкой сухостью во рту с сохранением реакции на стимуляцию, а 2-й степени умеренной сухостью во рту со слабой реакцией на стимуляцию. Для 3-й степени повреждений характерны полная сухость полости рта и отсутствие реакции на стимуляцию. Четвертая степень ксеростомии проявлялась фиброзом слюнных желез.

Выявлены значительные различия в частоте поздних лучевых повреждений кожи, подкожной клетчатки и слюнных желез у пациентов двух исследуемых групп $(<0,001)$.

Пациенты контрольной группы чаще всего имели поздние лучевые реакции кожи $1-2$ степени (50,4 и 39,2\%, соответственно). Большинство пациентов основной группы имели повреждения кожных покровов 1-й степени (75\%), в 21\% случаев кожных изменений не наблюдалось (рис. 4).

Похожая картина наблюдалась при изучении поздних лучевых реакций подкожной клетчатки - для контрольной группы были характерны повреждения 1-2 степени (48,8 и 42,4\%, соответственно), для основной - 1-я степень реакций $(52,4 \%)$. Повреждения 3 и 4 степени выраженности развивались в контрольной группе, соответственно, в 4 и в 0,8\% случаев, в отличие от основной группы, где они не были выявлены (рис. 5).

При изучении поздних лучевых реакций слюнных желез ни в одной группе не наблюдалось изменений 4-й степени выраженности. В контрольной группе чаще развивалась ксеростомия 1 и 2 степеней, соответственно, в 40,8 и 33,6\% случаев. В основной группе у 47,6\% пациентов зафиксирована 1-я степень лучевых изменений, 2-я степень наблюдалась у 26,6\% пациентов. Не выявлены проявления ксеростомии у $12 \%$ пациентов контрольной группы и у 25\% пациентов группы с нетрадиционным фракционированием дозы (рис. 6).

\section{Выводы}

Применение режима динамического ускоренного гиперфракционирования с со- 


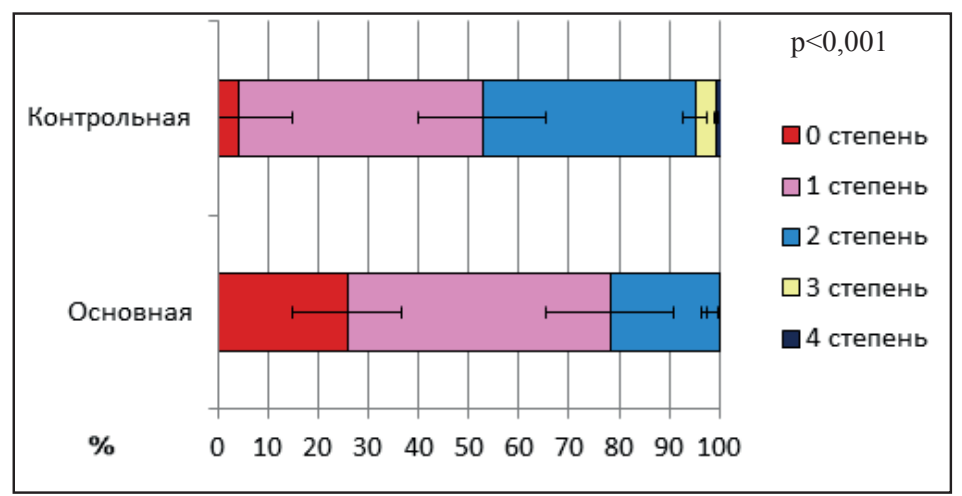

Рисунок 5. - Поздние лучевые повреждения подкожной клетчатки у пациентов контрольной и основной групп

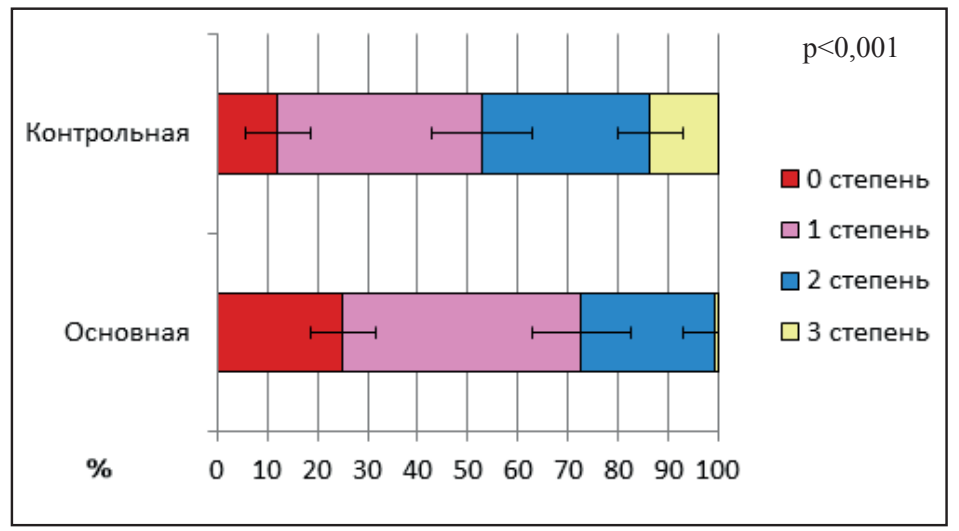

Рисунок 6. - Поздние лучевые реакции слюнных желез у пациентов контрольной и основной групп

путствующим бустом в рамках химиолучевого лечения позволило сократить частоту и степень

\section{Лumepamypa}

1. An intergroup phase III comparison of standard radiation therapy and two schedules of concurrent chemoradiotherapy in patients with unresectable squamous cell head and neck cancer / D. J. Adelstein [et al.] // J. Clin. Oncol. - 2003. - Vol. 21, № 1. - P. 92-98. - doi: 10.1200/ JCO.2003.01.008.

2. Concomitant chemoradiotherapy versus acceleration of radiotherapy with or without concomitant chemotherapy in locally advanced head and neck carcinoma (GORTEC 9902): An open-label phase 3 randomised trial / J. Bourhis [et al.] // Lancet Oncol. - 2012. - Vol. 13, № 2. - P. 145153. - doi: 10.1016/S1470-2045(11)70346-1.

3. Concurrent chemotherapy and radiotherapy for organ preservation in advanced laryngeal cancer / A. A. Forastiere [et al.] // N. Engl. J. Med. - 2003. - Vol. 349, № 22. - P. 2091-2098. - doi: 10.1056/ NEJMoa031317.

4. Concomitant cisplatin significantly improves locoregional control in advanced head and neck cancers treated with hyperfractionated radiotherapy / P. Huguenin [et al.] // J. Clin. Oncol. - 2004. - Vol. 22, № 23. - P. 4665-4673. doi:10.1200/JCO.2004.12.193.

5. Hyperfractionated or accelerated radiotherapy in head and neck cancer: A meta-analysis / J. Bourhis [et al.] // Lancet. - 2006. - Vol. 368, № 9538. - P. 843-854. - doi: 10.1016/ S0140-6736(06)69121-6. выраженности ранних лучевых реакций слизистых оболочек и кожи благодаря уменьшению общего времени лучевой терапии на 2 недели. Гематологическая токсичность в обеих группах оказалась сопоставима, что говорит о большем вкладе химиотерапии для ее развития, нежели лучевого компонента с разными вариантами фракционирования дозы.

Полученные с помощью линейно-квадратичной модели теоретические данные о более низкой биологически эффективной дозе и суммарной эквивалентной дозе при разработанном режиме фракционирования в отличие от стандартного режима подтверждены клиническими данными - поздние лучевые реакции кожи, подкожной клетчатки и слюнных желез развивались значимо реже в основной группе. Ранние лучевые реакции нормальных тканей и органов в группе с нетрадиционным режимом фракционирования не успевали развиться до тяжелой степени за более короткий период облучения по сравнению с конвенциональной семинедельной лучевой терапией.

\section{Заключение}

Полученные данные рандомизированного клинического исследования свидетельствуют об удовлетворительной переносимости одновременной химиолучевой терапии с нетрадиционным режимом фракционирования по критериям ранней и поздней токсичности в сравнении со стандартным курсом химиолучевого лечения.

6. A Radiation Therapy Oncology Group (RTOG) phase III randomized study to compare hyperfractionation and two variants of accelerated fractionation to standard fractionation radiotherapy for head and neck squamous cell carcinomas: first report of RTOG 9003 / K. K. Fu [et al.] // Int. J. Radiat. Oncol. Biol. Phys. - 2000. -Vol. 48, № 1. P. 7-16.

7. Katori, H. Comparison of hyperfractionation and conventional fractionation radiotherapy with concurrent docetaxel, cisplatin and 5-fluorouracil (TPF) chemotherapy in patients with locally advanced squamous cell carcinoma of the head and neck (SCCHN) / H. Katori, M. Tsukuda, K. Watai // Cancer Chemother. Pharmacol. - 2007. - Vol. 60, № 3. - P. 399-406. - doi: 10.1007/ s00280-006-0370-y.

8. Five compared to six fractions per week of conventional radiotherapy of squamous-cell carcinoma of head and neck: DAHANCA 6\&7 randomised controlled trial / J. Overgaard [et al.] // Lancet. - 2003. - Vol. 362, № 9388. - P. 933-940.

9. Pajonk, F. Radiation resistance of cancer stem cells: the 4 R's of radiobiology revisited / F. Pajonk, E. Vlashi, W. H. McBride // Stem Cells. - 2010. - Vol. 28, №4. P. 639-648. - doi: 10.1002/stem.318.

10. Линейно-квадратичная модель и фракционирование дозы при лучевой терапии злокачественных опухолей головы и шеи / Б. П. Афанасьев [и др.] // Медицинская 
радиология и радиационная безопасность. - 2009. T. 54, № 6. - С. 57-64.

\section{References}

1. Adelstein DJ, Li Y, Adams GL, Wagner H Jr, Kish JA, Ensley JF, Schuller DE, Forastiere AA. An intergroup phase III comparison of standard radiation therapy and two schedules of concurrent chemoradiotherapy in patients with unresectable squamous cell head and neck cancer. J. Clin. Oncol. 2003;21(1):92-98. doi: 10.1200/ JCO.2003.01.008.

2. Bourhis J, Sire C, Graff P, Grégoire V, Maingon P, Calais G, Gery B, Martin L, Alfonsi M, Desprez P, Pignon T, Bardet E, Rives M, Geoffrois L, Daly-Schveitzer N, Sen S, Tuchais C, Dupuis O, Guerif S, Lapeyre M, Favrel V, Hamoir M, Lusinchi A, Temam S, Pinna A, et al. Concomitant chemoradiotherapy versus acceleration of radiotherapy with or without concomitant chemotherapy in locally advanced head and neck carcinoma (GORTEC 99-02): An open-label phase 3 randomised trial. Lancet Oncol. 2012;13(2):145-153. doi: 10.1016/S14702045(11)70346-1.

3. Forastiere AA, Goepfert H, Maor M, Pajak TF, Weber R, Morrison W, Glisson B, Trotti A, Ridge JA, Chao C, Peters G, Lee DJ, Leaf A, Ensley J, Cooper J. Concurrent chemotherapy and radiotherapy for organ preservation in advanced laryngeal cancer. N. Engl. J. Med. 2003;349(22):2091-2098. doi: 10.1056/NEJMoa031317.

4. Huguenin P, Beer KT, Allal A, Rufibach K, Friedli C, Davis JB, Pestalozzi B, Schmid S, Thöni A, Ozsahin M, Bernier J, Töpfer M, Kann R, Meier UR, Thum P, Bieri S, Notter M, Lombriser N, Glanzmann C. Concomitant cisplatin significantly improves locoregional control in advanced head and neck cancers treated with hyperfractionated radiotherapy. J. Clin. Oncol. 2004;22(23):46654673. doi: 10.1200/JCO.2004.12.193.

5. Bourhis J, Overgaard J, Audry H, Ang KK, Saunders M, Bernier J, Horiot JC, Le Maotre A, Pajak TF, Poulsen MG,
O’Sullivan B, Dobrowsky W, Hliniak A, Skladowski K, Hay JH, Pinto LH, Fallai C, Fu KK, Sylvester R, Pignon JP; Meta-Analysis of Radiotherapy in Carcinomas of Head and neck (MARCH) Collaborative Group. Hyperfractionated or accelerated radiotherapy in head and neck cancer: A meta-analysis. Lancet. 2006;368(9538):843-854. doi: 10.1016/S0140-6736(06)69121-6.

6. Fu KK, Pajak TF, Trotti A, Jones CU, Spencer SA, Phillips TL, Garden AS, Ridge JA, Cooper JS, Ang KK. A Radiation Therapy Oncology Group (RTOG) phase III randomized study to compare hyperfractionation and two variants of accelerated fractionation to standard fractionation radiotherapy for head and neck squamous cell carcinomas: first report of RTOG 9003. Int. J. Radiat. Oncol. Biol. Phys. 2000;48(1):7-16.

7. Katori H, Tsukuda M, Watai K. Comparison of hyperfractionation and conventional fractionation radiotherapy with concurrent docetaxel, cisplatin and 5-fluorouracil (TPF) chemotherapy in patients with locally advanced squamous cell carcinoma of the head and neck (SCCHN). Cancer Chemother. Pharmacol. 2007;60(3):399-406. doi: 10.1007/s00280-006-0370-y.

8. Overgaard J, Hansen HS, Specht L, Overgaard M, Grau C, Andersen E, Bentzen J, Bastholt L, Hansen O, Johansen J, Andersen L, Evensen JF. Five compared to six fractions per week of conventional radiotherapy of squa-mous-cell carcinoma of head and neck: DAHANCA 6\&7 randomised controlled trial. Lancet. 2003;362(9388):933-940.

9. Pajonk F, Vlashi E, McBride WH. Radiation resistance of cancer stem cells: the 4 R's of radiobiology revisited. Stem Cells. 2010;28(4):639-648. doi: 10.1002/stem.318.

10. Afanasev BP, Akimov AA, Kozlov AP, Ushakova GA. Linejno-kvadratichnaja model i frakcionirovanie dozy pri luchevoj terapii zlokachestvennyh opuholej golovy i shei [The linear-quadratic model and dose fractionation at radiation therapy of head and neck cancers]. Medicinskaja radiologija i radiacionnaja bezopasnost [Medical radiology and radiation safety]. 2009;54(6):57-64.

\title{
ACUTE AND LATE TOXICITY WITH CHEMORADIOTHERAPY FOR OROPHARYNGEAL CANCER DEPENDING ON THE FRACTIONATION OPTION
}

\author{
Parkhomenko L. B.
}

\author{
Belorussian Medical Academy of Postgraduate Education, Minsk, Belarus
}

Background: Chemoradiotherapy fractionation regimen has been developed to reduce the frequency and severity of acute and late toxicity of normal tissues.

Purpose: To reduce the incidence and severity of acute and late toxicity in patients with cancer of the oropharyngeal zone stages III-IVA, B by using chemoradiotherapy with dynamic accelerated hyperfractionation with concomitant boost.

Material and methods: The data of 280 patients with squamous cell carcinoma of the oral cavity, tongue, oropharynx and hypopharynx stages III-IVA, B were analyzed. In the main group the altered fractionation radiotherapy was conducted. In the control group conventional radiotherapy was used. A comparative evaluation of the effectiveness of radiotherapy with conventional and altered fractionation regimens was conducted.

Results of research: In the main group, grade $2(63,6 \%)$ acute oral mucosa toxicity predominated, and in the control group grades $3(47,5 \%)$ and $2(41,6 \%)(p<0,001)$ were most common. Grade 1 acute skin toxicity was present in 53\% cases in the main group, while in the control group grade 2 skin reactions developed more often - 59,9\% $(p<0,001)$. Grade 1 late skin toxicity was more frequent in the main group - 75\% compared to the control group - 50\% $(p<0,001)$.

Conclusions: In the group with altered fractionation radiotherapy, a statistically significant decrease in the frequency and degree of toxicity was obtained.

Keywords: cancer of oropharyngeal zone, acute and late toxicity, altered fractionation radiotherapy. 